\title{
Inhalt, Vol. 11, No. 5, 1988
}

\section{Contents}

Impressum 198

Laudatio 200

Hölzel, D.; Saner, H.; de Waal, J. C. (München)

Tumornachsorgeschemata: Wissensinhalt - Anwendung -

Optimierung 202

Schenck, U.; Jutting, U.; Eiermann, W. (München)

Hormonrezeptorbestimmung beim Mammakarzinom mit

der Dextran-Coated-Charcoal-Methode und mit mono-

klonalen Antikörpern: Korrelation mit einem zytomor-

phologischen Grading 211

Schillínger, H.; Traeder, R.; Klosa, W.; Pohl, J. (Freiburg)

Nachweis von Lebermetastasen gynäkologischer Mali-

gnome durch Sonographie, Szintigraphie, Computer-

tomographie und Leberenzyme 216

Informationen für die Klinik 221

Wolf, M.; Havemann, K.; Stalleicken, D.; Gropp, C; Maasberg, M.; Braun, C. (Marburg); Hans, K. (Oberhausen); von Bülzingslöwen, F. (Donaustaufen); Klasen, H. (Oldenburg); Becker, H.; Häßler, R. (Fulda); Schroeder, M. (Duisburg); Hirschmann, H.; Gerdes, H. (Kassel); Hruska, E. (Gerlingen); Mende, S. (Ravens-burg); Pieritz, H. G. (Mayen); Holle, R. (Heidelberg) Ergebnisse zweier multizentrischer Therapiestudien beim inoperablen nichtkleinzelligen Bronchialkarzinom .... 222

Sonderbände 231

Schaefer, H.-J.; Ollenschläger, G.; Mödder, B. (Köln)

Orale Polychemotherapie bei weit fortgeschrittenen

Rezidiven des Morbus Hodgkin 233

Hinweise für Autoren 236

Kolaric, K.; Zupanc, D. (Zagreb/Jugoslawien);

Stahl, K. W,; Hinz, J.; Kempf, S. R.; Ivankovic, S.

(Heidelberg)

Verhinderung von Extravasatnekrosen als Komplikation

nach intravenöser Zytostatikatherapie. - Ergebnisse

einer offenen Pilotstudie 238

Paasen, A.; Stimmel, A.; Neumann, H. A. (Bochum)

Übertragung von INMEDD-Dateien auf einen Perso-

nalcomputer. Ein neues Konzept zur Flexibilisierung des

INMEDD 242

Imprint . 198 
Laudatio 200

Hölzel, D.; Saner, H.; de Waal, J. C. (Munich)

Follow-up of Tumor Patients: Criteria for Timing and

Test Selection 202

Schenck, U.; Jutting, U.; Eiermann, W. (Munich)

Cytomorphological Grading and Hormone Receptor

Status of Breast Cancer Determined with the Dextran-

Coated Charcoal Method and with Monoclonal Anti

bodies: Visual Examination of Biopsy Smears 211

Schillinger, H.; Traeder, R.; Klosa, W.; Pohl, J.

(Freiburg)

Sonography, Computed Tomography, Scintigraphy and

Liver Enzymes in the Diagnosis of Liver Metastasis in

Gynecological Tumors 216

Clinical Information 221

Wolf, M.; Havemann, K.; Stalleicken, D.; Gropp, C; Maasberg, M.; Braun, C. (Marburg); Hans, K. (Oberhausen); von Bülzingslöwen, F. (Donaustaufen); Klasen, H. (Oldenburg); Becker, H.; Häßler, R. (Fulda); Schroeder, M. (Duisburg); Hirschmann, H.; Gerdes, H. (Kassel); Hruska, E. (Gerlingen); Mende, S. (Ravens-burg); Pieritz, H. G. (Mayen); Holle, R. (Heidelberg) Two Multicenter Trials of Chemo- and Radiotherapy for Non-resectable Non-Small Cell Lung Cancer 222

Special Editions 231

Schaefer, H.-J.; Ollenschläger, G.; Mödder, B. (Cologne)

Oral Third-Line Salvage Therapy in Heavily Pretreated

Hodgkin's Disease 233

Instructions to Authors 236

Kolaric, K.; Zupanc, D. (Zagreb/Jugoslavia);

Stahl, K. W.; Hinz, J.; Kempf, S. R.; Ivankovic, S.

(Heidelberg)

Prevention of Extravasation Necroses Following

Intravenous Cytostatic Therapy. Results of an Open Pilot

Study 238

Paasen, A.; Stimmel, A.; Neumann, H. A. (Bochum)

Transmission of INMEDD Data to a Personal Computer.

A New Concept for Increasing the Flexibility of

INMEDD 242

Bibliographischer Hinweis: Inhaltsverzeichnisse dieser Zeitschrift erscheinen regelmäßig in current contents ${ }^{\circledR}$ sowie in anderen bibliographischen Diensten. 